refer to Fig. 1, which shows clearly that both for $y_{0}\left(y_{0}^{\prime}\right)$ greater and smaller than $y_{0}(.7862)$, we will have $\psi_{0} \delta y_{0}>0$, i.e., $\delta D>0$.

\title{
REFERENCES
}

1. G. A. Bliss, Lectures on the calculus of variations, University of Chicago Press, 1947

2. O. Bolza, Lectures on the calculus of variations, University of Chicago Press, 1904

3. G. A. Bliss, Calculus of variations, Open Court Co., 1925

4. H. and B. S. Jeffreys, Mathematical physics, Cambridge University Press, 1950

5. H. S. Tan, On superaerodynamic drag, and its minimization, to be published

6. H. S. Tan, On optimum nose curves for superaerodynamic missiles, J. Aeronaut. Sci. 25, 263 (1958)

\section{NOTE ON THE SOLUTION OF THE NEUTRON DIFFUSION PROBLEM BY AN IMPLICIT NUMERICAL METHOD*}

By GEORGE A. BAKER, JR. (Los Alamos Scientific Laboratory, Los Alamos, New Mexico)

It is of interest that the implicit, numerical method of Baker and Oliphant [1, 2] for the solution of time-dependent heat-flow problems in rectangular regions may be usefully extended to obtain the solution of the time-independent neutron-diffusion equation

$$
\nabla D(r) \nabla \varphi-\chi(r) \varphi+S(r)=0
$$

for the neutron density $\varphi$. In the original work of [1], to obtain an accurate solution, even asymptotically, it was necessary to take $\Delta t$ small. This choice was necessary because of the occurrence of a term in a higher order space derivative multiplied by $\Delta t$. We will herein make a slight modification to remove this defect. Let us rewrite (1) as (for the two-dimensional case)

$$
\begin{aligned}
\beta \varphi+\nabla^{2} \varphi+\frac{1}{\beta} \frac{\partial^{4} \varphi}{\partial x^{2} \partial y^{2}}=\alpha^{-2} \frac{\partial \varphi}{\partial t} & +\beta \varphi+\frac{1}{\beta} \frac{\partial^{4} \varphi^{*}}{\partial x^{2} \partial y^{2}} \\
& +\left(\frac{\chi(r)}{D(r)}-\mu\right) \varphi^{*}+\mu \varphi-\frac{S(r)}{D(r)}+\frac{\nabla D \cdot \nabla \varphi^{*}}{D(r)}
\end{aligned}
$$

where

$$
\varphi=\varphi^{*}, \quad \frac{\partial \varphi}{\partial t}=0
$$

and

$$
\mu=\operatorname{Min}_{r}[\chi(r) / D(r)] \text {. }
$$

To obtain the solution of (1) via (2) and (3) we must advance the time until the asymptotic solution of (2) is obtained. In [1], we described for the special case, $D$, a constant, how to guess $\varphi^{*}$, and then use (2) to calculate $\varphi_{\text {calc }}$. by solving the left-hand side and then how to compute a new guess, $\varphi^{* *}$, by means of

*Received September 30, 1958. Work performed under the auspices of the United States Atomic Energy Commission. 


$$
\varphi^{* *}=\frac{(F+\omega-1) \varphi^{*}+\varphi_{\mathrm{cal0}} .}{F+\omega}
$$

where

$$
\begin{aligned}
& \lambda=\frac{-3 \alpha^{-2}}{2 \Delta t}, \beta=\lambda-\mu, \quad \omega=\lambda / \beta, \\
& F=-\chi(r) /[D(r) \beta], \quad W=-S(r) /[D(r) \beta] .
\end{aligned}
$$

In [1] this process was continued until $\varphi^{* *}$ converged within a desired accuracy to $\varphi$. For the steady state, if we obtain $\varphi^{*}$ by linear extrapolation from the two previous times, then the first $\varphi^{* *}$ obtained is a sufficiently good approximation for $\varphi$ and one can proceed directly to the next time step.

If we assume that $D(r)$ half-way between mesh points is obtained by averaging the reciprocals (for conservation of flux at discontinuities), then the proper difference equation representation for $\beta(\Delta x)^{2}(\Delta y)^{2}$ times the right-hand side of (2) would be

$$
\begin{aligned}
& \beta(\Delta x)^{2} \beta(\Delta y)^{2}\left\{W(i, j)+\omega\left[^{\frac{4}{3}}{ }^{1} \varphi(i, j)-\frac{1}{3}^{2} \varphi(i, j)\right]-[F(i, j)+\omega-1] \varphi^{*}(i, j)\right\} \\
& -\beta(\Delta y)^{2}\left\{\left[\frac{D(i+1, j)-D(i, j)}{D(i+1, j)+D(i, j)}\right]\left[\varphi^{*}(i+1, j)-\varphi^{*}(i, j)\right]\right. \\
& \left.+\left[\frac{D(i, j)-D(i-1, j)}{D(i, j)+D(i-1, j)}\right]\left[\varphi^{*}(i, j)-\varphi^{*}(i-1, j)\right]\right\} \\
& -\beta(\Delta x)^{2}\left\{\left[\frac{D(i, j+1)-D(i, j)}{D(i, j+1)+D(i, j)}\right]\left[\varphi^{*}(i, j+1)-\varphi^{*}(i, j)\right]\right\} \\
& +\left[\frac{D(i, j)-D(i, j-1)}{D(i, j)+D(i, j-1)}\right]\left[\varphi^{*}(i, j)-\varphi^{*}(i, j-1)\right] \\
& +4 \varphi^{*}(i, j)-2\left[\varphi^{*}(i+1, j)+\varphi^{*}(i, j+1)\right. \\
& \left.+\varphi^{*}(i-1, j)+\varphi^{*}(i, j-1)\right]+\varphi^{*}(i+1, j+1) \\
& +\varphi^{*}(i+1, j-1)+\varphi^{*}(i-1, j+1)+\varphi^{*}(i-1, j-1) \text {, }
\end{aligned}
$$

where ${ }^{1} \varphi$ is $\varphi$ at $t-\Delta t$ and ${ }^{2} \varphi$ is $\varphi$ at $t-2 \Delta t$.

In order to obtain the asymptotic solution as quickly as possible, we would like to choose $\Delta t$ as large as possible. Hence we wish to pick $-\beta$ as small as possible. However, as was pointed out in [1], the method loses about [- $\left.\log _{2}\left(0.2 \beta^{2}(\Delta x)^{2}(\Delta y)^{2}\right)\right]$ binary bits due to the cancellation of nearly equal numbers. Therefore, if we carry eight decimal places and wish to retain four figure accuracy, we must restrict

$$
\beta^{2}(\Delta x)^{2}(\Delta y)^{2} \geq 5 \times 10^{-4} \text {. }
$$

As a general rule, our experience has shown that about 5 to 10 iterations are required and the choice of a smaller $\beta$ than that needed to assure convergence in this number of iterations does not speed up the convergence. We find

$$
\beta(\Delta x)^{2} \text { and } \beta(\Delta y)^{2} \sim-(\mu+50 / N),
$$

where $N$ is the number of mesh points, to be a good rough guide to the size of $\beta$ to be used, subject of course to (8). 
When $D(r)$ is not constant, $\beta$ is subject to another restriction besides (8). If we represent $\varphi^{*}, \varphi_{\text {oalo. }}$, and $\varphi^{* *}$ as $\varphi$ plus an error term, it is easy to show using the method of Sec. 3 of [1] that if $\epsilon^{*}$ is the error in $\varphi^{*}$, then the error in $\varphi^{* *}, \epsilon^{* *}$, is to first order approximately

$$
\epsilon^{* *}=-\frac{\nabla D \cdot \nabla \epsilon^{*}}{\beta D(F+\omega)} .
$$

From (7) it is evident that the $x$, and $y$ components of $(\nabla D / D)$ are bounded in magnitude by $(\Delta x)^{-1}$ and $(\Delta y)^{-1}$ respectively. The term $F+\omega$ has a minimum value of unity. At a discontinuity $(\Delta x$ or $\Delta y) \nabla \epsilon^{*}$ may well be of the order of $\epsilon^{*}$, hence we see $-\beta(\Delta x)^{2}$ and $-\beta(\Delta y)^{2}$ must be at least unity. We find in practice that a satisfactory choice of $\beta$ may be obtained from the rule that $-\beta(\Delta x)^{2}$ and $-\beta(\Delta y)^{2}$ are greater than 2 . If the discontinuities in $D$ are slight then we can reduce the value of $\beta$ correspondingly. It. seems desirable to provide a convergence factor of about $\frac{1}{2}$ between $\epsilon^{*}$ and $\epsilon^{* *}$ in (10).

In the problems $(N \lesssim 2000)$ we have run using this method, most took about 7 to 15 iterations to reduce the error by a factor of 100 . Large problems with sharp discontinuities: in $D$ can take considerably longer, due to the restriction imposed by (10) on $\beta$.

\section{REFERENCES}

1. G. A. Baker, Jr. and T. A. Oliphant, An implicit, numerical method for solving the two dimensional heat equation, to appear in Quart. Appl. Math.

2. G. A. Baker, Jr., An implicit, numerical method for solving the n-dimensional heat equation, to appear in Quart. Appl. Math.

\section{FUNCTIONAL EQUATIONS AND MAXIMUM RANGE*}

\section{BY RICHARD BELLMAN (The RAND Corporation)}

1. Introduction. The current interest in rockets and space travel has aroused a corresponding interest in the determination of maximum range, minimum time, and so on, for various types of trajectories.

A variety of questions of this type have been treated by means of the theory of dynamic programming, see $[1,2,4]$. Here we wish to show how to use functional equations to determine the range, the maximum elevation, and similar quantities, as functions of initial position and velocities.

2. Vertical motion-I. Consider an object, subject only to the force of gravity and the resistance of the air, which is propelled straight up. In order to illustrate the technique we shall employ, let us treat the problem of determining the maximum altitude.

Let the defining equation be

$$
u^{\prime \prime}=-g-h\left(u^{\prime}\right),
$$

with the initial conditions $u(0)=0, u^{\prime}(0)=v$. Here $v>0$, and $h\left(u^{\prime}\right) \geq 0$ for all $u^{\prime}$.

Since the maximum altitude is a function of $v$, let us introduce the function

$$
f(v)=\text { the maximum altitude attained starting with initial velocity } v \text {. }
$$

\footnotetext{
*Received October 20, 1958.
} 